\title{
Video Article \\ Application of Laser Micro-irradiation for Examination of Single and Double Strand Break Repair in Mammalian Cells
}

\author{
Nathaniel W Holton ${ }^{1}$, Joel F Andrews ${ }^{1}$, Natalie R Gassman ${ }^{1}$ \\ ${ }^{1}$ Department of Oncologic Sciences, University of South Alabama Mitchell Cancer Institute \\ Correspondence to: Natalie R Gassman at nrgassman@health.southalabama.edu \\ URL: https://www.jove.com/video/56265 \\ DOI: doi:10.3791/56265
}

Keywords: Cancer Research, Issue 127, DNA repair, laser micro-irradiation, DNA damage, strand break, XRCC1, gamma-H2AX, strand break signaling

Date Published: 9/5/2017

Citation: Holton, N.W., Andrews, J.F., Gassman, N.R. Application of Laser Micro-irradiation for Examination of Single and Double Strand Break Repair in Mammalian Cells. J. Vis. Exp. (127), e56265, doi:10.3791/56265 (2017).

\section{Abstract}

Highly coordinated DNA repair pathways exist to detect, excise and replace damaged DNA bases, and coordinate repair of DNA strand breaks. While molecular biology techniques have clarified structure, enzymatic functions, and kinetics of repair proteins, there is still a need to understand how repair is coordinated within the nucleus. Laser micro-irradiation offers a powerful tool for inducing DNA damage and monitoring the recruitment of repair proteins. Induction of DNA damage by laser micro-irradiation can occur with a range of wavelengths, and users can reliably induce single strand breaks, base lesions and double strand breaks with a range of doses. Here, laser micro-irradiation is used to examine repair of single and double strand breaks induced by two common confocal laser wavelengths, $355 \mathrm{~nm}$ and $405 \mathrm{~nm}$. Further, proper characterization of the applied laser dose for inducing specific damage mixtures is described, so users can reproducibly perform laser microirradiation data acquisition and analysis.

\section{Video Link}

The video component of this article can be found at https://www.jove.com/video/56265/

\section{Introduction}

Fluorescent microscopy has emerged as a powerful technique to visualize cellular architecture, examine protein localization, and monitor protein-protein and protein-DNA interactions. Using fluorescent microscopy to study DNA damage responses after the application of global DNA damaging agents, such as ultraviolet (UV) light, ionizing radiation, chemical oxidizing or alkylating agents, and/or chemotherapeutics has provided new insight into the initiation, signaling, and recruitment of DNA repair proteins to sites of DNA damage ${ }^{1,2}$. However, these global and asynchronous damaging events are limiting, if detailed information about the recruitment order, kinetics of association or dissociation, and the relationships between key DNA repair proteins are sought. Fortunately, advancements in laser scanning confocal microscopes, broader availability of damage-inducing laser wavelengths, and improvements in fluorescent proteins over the past 25 years have provided researchers with improved tools for examining these aspects of DNA repair, through targeted DNA damage induction.

Irradiation of cells with laser microbeams in order to study cellular and subcellular functions is a well-established tool in cell and radiation biology ${ }^{3}$. Application of this technique to the study of DNA repair emerged when Cremer and co-workers used a highly-focused UV ( $257 \mathrm{~nm}$ ) laser microbeam system to induce DNA damage over a $0.5 \mu \mathrm{m}$ spot in Chinese hamster ovary (CHO) cells ${ }^{4}$ and established the induction of DNA photolesions by this system ${ }^{5}$. While offering significant improvements over UV microbeam methods at the time, adoption of this damaging system was limited due to its specialized set up, and its inability to generate double strand breaks (DSBs) ${ }^{6}$. Subsequent investigation of a variety of UV-B (290 - $320 \mathrm{~nm})$ and UV-A (320 - $400 \mathrm{~nm}$ ) wavelengths by a number of groups revealed that UV photoproducts, oxidative base lesions, single strand breaks (SSBs), and DSBs could be induced dependent on the laser wavelength and power applied ${ }^{4,7,8,9,10}$ (reviewed in ${ }^{3}$ ). Further, combinations of these UV-B and UV-A wavelengths with sensitizing agents, like psoralen, bromodeoxyuridine (BrdU), and Hoechst dyes, were also found to induce DNA damage dependent on wavelength, power, and duration of exposure, though the power needed to induce damage is often lowered in the presence of these agents ${ }^{11,12,13,14,15}$. These advancements expanded the use of micro-irradiation, though there were still technical hurdles to be addressed for wider adoption of these methods.

Cremer and co-workers significantly advanced the field of micro-irradiation by precisely focusing the UV microbeam to apply significant damaging energy over a highly localized area in the cell. As confocal microscopes and laser microdissection systems advanced, tightly focused light was more broadly accessible; however, coupling UV sources to scopes and dealing with the chromatic aberrations they induced still presented significant challenges to most users ${ }^{3,6,16}$. As UV dyes increased in popularity throughout the 1990s, optics capable of focusing and capturing UV-excited fluorescence became more widely available ${ }^{16}$, and improvements in laser scanning offered users the ability to create highly focused UV excitation spots within cells ${ }^{6,17}$. However, it was not until the early 2000 s that the true impact of this combination of tightly focused beams with higher intensity lasers was felt, when numerous reports emerged demonstrating that DNA strand breaks could be induced with and without sensitizers in the UV-A range ${ }^{6,10,18,19,20}, 405 \mathrm{~nm}^{21,22,23,24,25,26}$, and even at longer visible wavelengths like $488 \mathrm{~nm}^{27}$. These advancements allowed for more widespread adoption of the micro-irradiation technique on a number of commercial systems. In parallel with 
these developments, two-photon techniques also emerged that allowed precise induction of DNA damage; though these advancements will not be discussed here, there are a number of review articles discussing these methodologies ${ }^{9,28,29,30}$.

With the current accessibility of confocal microscopes capable of delivering highly focused UV light and the wide-spread availability of fluorescent proteins to allow real-time tracking of DNA repair proteins, micro-irradiation techniques have evolved into powerful tools for examining DNA damage response and repair pathways. However, users need to be aware that the generation of DNA damage is highly dependent on the laser wavelength and the power applied to the sub-nuclear region. Use of UV-C ( 260 nm) wavelengths allow direct DNA excitation and high selectivity for the induction of UV photoproducts ${ }^{7,8}$. UV-B and UV-A wavelengths produce mixtures of DNA damage (base lesion, SSBs, and DSBs), dependent on the applied power and the cellular background utilized ${ }^{\text {}}$. Endogenous photosensitizers and anti-oxidant levels in the targeted cells can influence the DNA damage mixtures produced by these wavelengths. Additionally, the use of exogenous photosensitizers (BrdU, etc.) may assist in lowering the energy needed for the induction of DNA damage. However, these agents can induce DNA damage by themselves, and they may alter the cell cycle and chromatin structure, so their use may produce undesired effects that need to be considered by the user. Therefore, prior to using micro-irradiation to study DNA damage response and repair, careful consideration of the DNA repair pathway of interest, the wavelengths available for use, and the DNA damage mixture created is required.

Here, laser micro-irradiation is performed at two commonly used wavelengths, $355 \mathrm{~nm}$ and $405 \mathrm{~nm}$, without sensitizers to demonstrate the mixtures of DNA damage induced by these wavelengths and the influences these damage mixtures have on examining the repair of SSBs and DSBs. Users should be aware that these wavelengths do not create a single species of strand breaks or base lesions. In order to discriminate between DNA repair pathways, users must carefully control the applied power over a specific region of the nucleus and characterize the induced damage using multiple strand break markers and DNA lesion antibodies. If properly applied and characterized, laser micro-irradiation can enrich some species of DNA damage, allowing users to assess repair of base lesions and SSBs or DSBs, with some specificity. Therefore, we have provided a method allowing users to reproducibly perform laser micro-irradiation, characterize the DNA damage mixtures induced by the applied laser dose, and perform data analysis.

\section{Cell Culture and generation of stable cells}

1. Grow $\mathrm{CHO}-\mathrm{K} 1$ cells in minimal essential medium supplemented with $10 \%$ fetal bovine serum. Maintain cells in a humidified incubator with $5 \%$ $\mathrm{CO}_{2}$ at $37^{\circ} \mathrm{C}$

2. Transfect $\mathrm{CHO}-\mathrm{K} 1$ cells with $1 \mu \mathrm{g}$ of plasmid DNA containing human XRCC1 with a C-terminal green fluorescent protein (GFP) tag using a commercial transfection reagent following the manufacturer's instructions.

3. Select CHO-K1 cells stably expressing the human XRCC1-GFP fusion using $800 \mu \mathrm{g} / \mathrm{mL}$ geneticin and enrich the XRCC1-GFP expressing population using fluorescence assisted cell sorting (FACS).

4. Plate cells to be micro-irradiated in culture vessels with coverglass bottoms, so they reach approximately $75 \%$ or greater confluency on the following day. Some spaces between cells are desirable, particularly if using registration to return to the same image field (described in section 3.2).

\section{Microscope set-up}

1. Select a laser scanning confocal microscope equipped with suitable lasers and optics, and control software for micro-irradiation/ photostimulation. Presented experiments use a laser scanning confocal microscope that has been modified to include a $355 \mathrm{~nm}$ laser, fibercoupled to a galvanometer photoactivation miniscanner and controlled using the manufacturer's control and analysis software. This system can perform micro-irradiation in a user-designated region of interest (ROI) using the photoactivation miniscanner (355 $\mathrm{nm}$ laser) or the standard confocal galvanometer (405 nm laser).

2. Select the wavelength to be used for micro-irradiation, ensuring that all optical components in the micro-irradiation lightpath are suitable for the chosen wavelength.

NOTE: The presented system uses a 40× C-Apochromat (numerical aperture (NA) 1.2) oil immersion objective together with a UV filter cube for $355 \mathrm{~nm}$ micro-irradiation, and a $20 \times$ C-Apochromat (NA 0.75) dry objective using the standard confocal lightpath for $405 \mathrm{~nm}$ microirradiation. The $20 \times$ objective used in the setup is not compatible with the $355 \mathrm{~nm}$ wavelength; therefore we used the $40 \times$ for $355 \mathrm{~nm}$ only. Using the dry objective for damaging allows immunofluorescence protocols to be applied downstream without cleaning off immersion oil, which is why we utilize this objective when possible.

3. Configure the microscope for the micro-irradiation experiment. To maintain consistency between experiments, designate a standardized configuration of the microscope components to be used for each type of micro-irradiation. Save these settings as a preset within the microscope's operating software, if possible.

NOTE: In the presented system, cells are micro-irradiated and imaged using unidirectional scanning, a scan resolution of $1024 \times 1024$ pixels with 1x scan zoom, at a frame rate of 8 frames per second (fps), and with the pinhole set to approximately 4 Airy Units (AU), as determined for the $488 \mathrm{~nm}$ laser $(69 \mu \mathrm{m})$. This open pinhole setting was chosen to maximize the amount of light captured in each image, allowing the use of lower imaging laser powers and reducing photobleaching.

\section{Laser micro-irradiation}

1. Place the prepared culture dish, containing cells of interest, onto the microscope stage and lock securely into place.

1. If available, place chambered slide in a stage-top incubator and maintain at $37^{\circ} \mathrm{C}$ with $5 \% \mathrm{CO}_{2}$ during damage induction. This step is most important for live-cell timelapse imaging or long imaging sessions. Otherwise place cells on the microscope stage and perform irradiation at room temperature $\left(\sim 25^{\circ} \mathrm{C}\right)$, and then quickly return cells to an incubator at $37{ }^{\circ} \mathrm{C}$ with $5 \% \mathrm{CO}_{2}$. 
2. Register image fields to allow the researcher to return to the same location after performing fixation, staining, or other procedures. Perform registration of damaged fields using an encoded, automated microscope stage or cell culture coverglass vessels with an etched or labeled grid of $X Y$ locations.

1. If using software image registration, select a recognizable feature of the culture vessel (i.e., the corner of the coverglass or barrier between wells), collect an image, and record the XY location. This will allow for alignment and registration of the $X Y$ locations of selected fields following sample preparation.

2. If using manual registration, record grid or etched locations for each field manually, being careful to record the orientation and placement of the dish on the stage.

3. If no identifying features for the cell culture vessels are available, identify damaged fields by visual inspection of cell morphology and density. Take care to select fields with sufficiently distinct features to be recognizable during later imaging. NOTE: This can be time consuming to perform unless the orientation and area of the culture vessel is tightly restricted.

3. Select a field for micro-irradiation and focus the sample. For cells expressing fluorescently labeled proteins, select the focal plane with the maximal nuclear cross-section in the fluorescent channel of interest. For cells not expressing labeled proteins, or for those without clear nuclear localization, phase contrast or differential interference contrast (DIC) imaging can be used to find the correct focal plane. NOTE: A useful feature of phase contrast and DIC imaging is the fact that as the focal plane moves through the sample, the same feature can appear light or dark depending on the relative focal plane.

1. Select a clear feature within the nucleus, such as a nucleolus, and move the focal plane up and down while observing this change in appearance. The true focal plane will lie within the transition from light to dark. To focus the sample, select the focal plane in which the selected feature has the sharpest contrast.

4. Register the position of the field of interest. Create a $3 \times 3$ pixel square ROI within the microscope software, place this ROI over the nucleus of a cell to be damaged, and set this ROI to be the damage ROI.

5. Collect a pre-damage image, including the position of the damage ROI.

1. For experiments that do not use fluorescent proteins, acquire a phase-contrast or differential interference contrast (DIC) brightfield image to identify and record nuclei for damage.

2. For live-cell experiments using fluorescent proteins, acquire an image containing brightfield and the fluorescence channel for the protein of interest (i.e., laser line $488 \mathrm{~nm}$ for excitation of GFP, laser line $561 \mathrm{~nm}$ for excitation of RFP). In experiments using the CHOK1 XRCC1-GFP, fluorescence excited by the $488 \mathrm{~nm}$ laser line was simultaneously collected with the transmitted DIC brightfield channel.

6. Initiate laser damage.

1. In the presented system, control the laser dose by the total time spent scanning the damage ROI. Because the galvanometer for the $355 \mathrm{~nm}$ laser operates at a fixed scan rate, laser dose is controlled by the time spent repeatedly scanning the selected damage ROI: in the data presented here, micro-irradiation at $355 \mathrm{~nm}$ is performed for 2 and 10 seconds.

2. In contrast, control the $405 \mathrm{~nm}$ laser dose by modulating the scan rate and perform one scan of the selected damage ROI. In the data presented here, use 8 and $0.5 \mathrm{fps}$ for micro-irradiation at $405 \mathrm{~nm}$. A scan rate of $8 \mathrm{fps}$ delivers a lower laser dose than $0.5 \mathrm{fps}$, because the laser spends less time on each pixel during the scan. Both lasers are operated at $100 \%$ power. See section 3.7 for instructions on measuring laser power directly.

NOTE: Each individual microscope system may differ in how laser power is delivered to a designated ROI, similar to how the $355 \mathrm{~nm}$ and $405 \mathrm{~nm}$ differ in the presented system. Users will need to determine this procedure for their microscope system and report these parameters within their methods sections.

3. For live cell imaging, perform timelapse image acquisition of brightfield and fluorescence channels. Adjust duration and frequency of timelapse to optimize data collection, ideally capturing the accumulation of the fluorescent protein at the damage ROI and its dissociation over the time course of the experiment. Experiments using XRCC1-GFP collected images every 30 seconds for 20 minutes.

NOTE: The frequency of the timelapse imaging can be limited by photobleaching of the fluorophore, complicating analysis of accumulation and dissociation. Photobleaching can be evaluated by observing undamaged cells during the timelapse and adjusting acquisition conditions to minimize fluorescent signal loss in these undamaged cells. Some photobleaching may be unavoidable, so users may compensate for the signal loss by normalizing the fluorescent intensities of damaged cells to those of undamaged cells in each frame of the timelapse.

1. After the time course is completed, select a new field of cells for damage or fix damaged cells for further analysis, as described in section 4

2. Continue micro-irradiation and timelapse imaging until the desired number of damaged cells is reached. NOTE: We recommend damaging a total of $10-25$ cells per selected condition in order to assess the cell-to-cell heterogeneity in response to the induced damage. Although most confocal systems will allow users to damage more than one cell during each micro-irradiation, this introduces staggered damage initiation times, which can complicate analysis of peak recruitment time over a large number of cells or dissociation time for fast events. In some systems, simultaneous damage induction over several ROls may reduce the laser dose received by each independent ROI. Therefore, unless these timing/power issues are directly addressed by the user, it is recommended that cells be damaged individually.

4. For analysis by immunofluorescence (IF), perform damage and either immediately fix cells, as described in section 4, or allow cells to repair for selected increments of time (i.e., 1, 5, 10 or $20 \mathrm{~min}$ ). After the desired time elapses, fix and stain the cells, as detailed in section 4.

1. To increase overall cell number for IF analysis, damage additional fields within the culture vessel to generate a multi-field time course of the post-damage response. Record the XY location of each field and the time that the damage occurred. After the desired total repair time elapses, fix and stain the cells as detailed below. 
7. After observing of protein recruitment at selected doses, measure and report laser power levels post-fiber and post-objective to accurately characterize and report laser doses. We use a compact digital power meter and two different photodiode sensor heads; one coupled directly to the laser fiber to measure post-fiber output, and the other placed on the microscope stage to measure post-objective output.

1. To measure laser power, place the sensor in the desired configuration (post-fiber or post-objective) and perform micro-irradiation as described above, while recording the measurements made by the power meter. Be aware that the sampling rate of the power meter may not be fast enough to capture very rapid micro-irradiation events, so it may be necessary to run multiple iterations of the experiment to ensure that the power meter accurately detects the applied dose.

NOTE: For the $355 \mathrm{~nm}$ laser, we measured an average peak power of approximately $5 \mathrm{~mW}$ post-fiber and approximately $19 \mu \mathrm{W}$ postobjective. For the $405 \mathrm{~nm}$ laser, micro-irradiation was performed in loops of 30 iterations to overcome the $0.01 \mathrm{~s}$ maximum sampling rate of the power meter, and average peak powers of $1.5 \mathrm{~mW}$ and $2.4 \mathrm{~mW}$ were measured using scan speeds of 8 and 0.5 frames per second, respectively. Every power meter is different. Users will need to determine the operational wavelengths and sampling rates for their individual power meter.

\section{Immunofluorescence staining procedures}

1. Analyze strand break induction by IF staining.

1. Fix cells with $3.7 \%$ formaldehyde (CAUTION) in phosphate buffered saline (PBS) for $10 \mathrm{~min}$. Aspirate formaldehyde solution and wash 3 times with PBS. The protocol can be stopped here by placing PBS back on the cells, and the cells can be stored at $4{ }^{\circ} \mathrm{C}$ for up to 1 week.

CAUTION: Formaldehyde is toxic and carcinogenic. Wear proper personal protective equipment and dispose of the toxic substance as instructed by institutional environmental health and safety procedures.

2. Permeabilize cells using $0.25 \%$ Triton $X-100$ in PBS for 10 min at room temperature (RT) and then wash 3 times with PBS.

3. Block non-specific antibody binding by incubating for $30 \mathrm{~min}$ in PBS containing $1 \%$ bovine serum albumin (BSA) at RT.

4. Incubate with primary monoclonal antibody against $\mathrm{YH} 2 \mathrm{AX}$ and primary polyclonal antibody against 53BP-1 both diluted $1: 750$ in $\mathrm{PBS}$ containing $1 \%$ BSA for $1 \mathrm{~h}$ at RT, and then wash 3 times with PBS.

5. Incubate cells with Alexa 488 goat anti-mouse and Alexa 546 goat anti-rabbit both diluted 1:2000 in PBS containing $1 \%$ BSA for $1 \mathrm{~h}$ at $\mathrm{RT}$, and then wash 3 times with PBS.

6. Stain nuclear DNA with a $10 \mathrm{mg} / \mathrm{mL}$ solution of DAPI (4',6-Diamidino-2-Phenylindole, CAUTION) diluted to 1:5000 in PBS for $5 \mathrm{~min}$, or use comparable nuclear dye, and then wash 3 times with PBS.

CAUTION: DAPI is toxic and mutagenic. Wear proper personal protective equipment and dispose the toxic substance as instructed by institutional environmental health and safety procedures.

7. Place PBS or PBS $+0.1 \%$ sodium azide (CAUTION) back onto the stained cells. Protocol can be stopped here and cells stored at $4{ }^{\circ} \mathrm{C}$ for several days, or proceed to image acquisition in section 5 .

CAUTION: Sodium azide is toxic. Wear proper personal protective equipment and dispose the toxic substances as instructed by institutional environmental health and safety procedures.

2. Analyze induction of base lesions or DNA adducts by IF staining

1. Fix and permeabilize cells in ice-cold methanol (CAUTION) for 20 min at $-20{ }^{\circ} \mathrm{C}$.

CAUTION: Methanol is toxic and flammable. Wear proper personal protective equipment and dispose the toxic substance as instructed by institutional environmental health and safety procedures.

2. Aspirate the methanol and allow the sample to dry completely for $15 \mathrm{~min}$. Protocol can be stopped here and cells stored at $-20{ }^{\circ} \mathrm{C}$ dry for up to 3 days.

3. Rehydrate the cells in PBS for 15 min.

4. Denature DNA using $2 \mathrm{~N} \mathrm{HCl}$ (CAUTION) for 45 min at RT and wash 3 times with PBS.

CAUTION: $\mathrm{HCl}$ is corrosive. Wear proper personal protective equipment and dispose the corrosive substance as instructed by institutional environmental health and safety procedures.

5. Neutralize in $50 \mathrm{mM}$ Tris- $\mathrm{HCl} \mathrm{pH} 8.8$ for $5 \mathrm{~min}$ followed by 3 washes with PBS.

6. Incubate cells in blocking buffer made with $5 \%$ normal goat serum and $0.1 \%$ Triton $\mathrm{X}-100$ in PBS for $1 \mathrm{~h}$ at RT.

7. Incubate with primary antibodies for 8-oxo-2'-deoxyguanosine (8-oxodG, 1:400) or cyclobutane pyrimidine dimer (CPD, 1:1,000) in the goat serum blocking buffer for $1 \mathrm{~h}$ at RT, and then wash 3 times with PBS.

8. Incubate cells with Alexa 488 goat anti-mouse at a 1:2000 ratio in the goat serum blocking buffer for $1 \mathrm{~h}$ at RT, and wash 3 times with PBS.

9. Stain nuclear DNA using 1:5000 DAPI (10 mg/mL) in PBS for $5 \mathrm{~min}$, and wash 3 times with PBS.

10. If using chambered coverglass, place PBS or PBS $+0.1 \%$ sodium azide back onto the stained cells. Protocol can be stopped here and cells stored at $4{ }^{\circ} \mathrm{C}$ for several days, or proceed to image acquisition in section 5 .

11. Alternatively, mount cells in preferred mounting medium, if an additional coverglass can be placed on top of the culture vessel. Once cured, mounted cells can be stored at $4{ }^{\circ} \mathrm{C}$ for long periods of time.

\section{Image acquisition for immunofluorescence experiments}

1. Clean the coverglass bottom of the culture vessel with ethanol and place it back on the confocal microscope stage. Ensure that the orientation and position of the vessel on the stage corresponds to the orientation and position recorded when damage was induced. Secure the culture vessel to ensure optimal registration and imaging.

2. Locate previously imaged fields using the registration technique selected (described in 3.2).

1. For manual registration using a coverglass with an etched grid, bring the grid into focus and find identifying marks. Then use recorded $\mathrm{XY}$ coordinates to locate damaged cells. 
2. For image-based automated registration, locate the structural feature used as a reference in step 3.2.1, and then align the current live microscope view as closely to the recorded image as possible. Once aligned, measure the current $X Y$ microscope stage location and compare it to the $X Y$ location of the reference image. The linear distance between the two locations defines the $X$ and $Y$ offset. Apply this offset to each recorded $X Y$ location to identify the image field(s) containing the irradiated cell. This offset function may be automated in the microscope control software or performed manually.

3. If no appropriate registration points could be identified, manually locate cells by scanning the slide and locating the previously identified cell features.

3. Acquire images using appropriate microscope settings to collect all stained targets, including a brightfield image (setting in section 2.3 and focusing in section 3.3). In presented experiments, multichannel images were collected using the following excitation laser lines and fluorophores: $405 \mathrm{~nm}$ (DAPI), $488 \mathrm{~nm}$ (Alexa 488 and transmitted DIC brightfield), and $561 \mathrm{~nm}$ (Alexa 546). All lasers pass through a single acousto-optical tunable filter controlling the transmission of both laser wavelength and power.

\section{Live cell image analysis of the recruitment of fluorescent proteins to micro-irradiated sites}

1. Open acquired images in an image analysis application (i.e., NIS Elements or ImageJ). If necessary, combine the pre-irradiation image with timelapse images to generate a single image sequence of pre- and post- damage induction. Users can show recruitment by measuring changes in fluorescent intensity over the irradiated area relative to the fluorescence intensity measured across the whole nucleus (see Representative Results Figure 1B).

2. For each cell to be measured, first generate a reference ROI that represents the nucleus. Use a thresholding algorithm on the fluorescent signal that contains the pixels making up the nucleus, and then convert this area into a ROI. If necessary, the ROI can be manually drawn in using the brightfield as a reference. Adjust the ROI over the time course to ensure the nuclear area is accurately covered by the ROI, and report the mean fluorescence intensity of the fluorescent signal within this ROI for each frame.

3. For each cell to be measured create a $6 \times 6$ pixel ROI and place it over the damage ROI for each frame of the time course. This larger ROI is now the damage ROI for analysis. Report the mean ROI fluorescence intensity for each frame. NOTE: Most commercial image analysis software contains modules for 2D object tracking, and there are a number of user-developed macros for ImageJ or FIJI that facilitate faster workflow and higher throughput (see https://imagej.nih.gov/ij/plugins/).

4. Normalize the mean damage ROI fluorescence intensity to that of a corresponding reference ROI in each frame of the timelapse. Here, the mean nuclear fluorescence intensity is used as the reference ROI (see Representative Results Figure 1). Normalization can be performed by subtracting the mean reference ROI fluorescence intensity from the mean damage ROI fluorescence intensity, or by dividing the mean damage ROI fluorescence intensity by the mean reference ROI fluorescence intensity. NOTE: Normalization by division can yield unpredictable results, if used in situations with very low reference intensity values.

5. Repeat for all damaged cells, as well as for at least two control, undamaged cells. Control cell results can be used for further normalization, if required.

6. Graph normalized intensity values over time to show changes in recruitment dynamics as a function of experimental treatment.

\section{Image analysis of protein recruitment detected by immunofluorescence}

1. Open pre- and post-damage images in an image analysis application. The pre-damage images contain the damage ROI(s) used for microirradiation. Copy the ROI(s) and paste into the post-damage images to identify targeted cells.

2. Generate a $6 \times 6$ pixel ROI for analysis of protein recruitment and place this ROI at the location of the damage ROI. This larger ROI is now the damage ROI for analysis. Report the mean damage ROI fluorescence intensity.

3. Normalize the mean damage ROI fluorescence intensity to that of a corresponding reference ROI. Here, the mean nuclear fluorescence intensity of the damaged cell is used as the reference ROI (see Representative Results Figure 1). Generate the reference ROI by using a thresholding algorithm on the DAPI signal to define the nucleus, and then convert this area to a ROI. Report the mean reference ROI fluorescence intensity. Normalization can be performed by subtracting the mean reference ROI fluorescence intensity from the mean damage $\mathrm{ROI}$ fluorescence intensity, or by dividing the mean damage ROI fluorescence intensity by the mean reference ROI fluorescence intensity. NOTE: Normalization by division can yield unpredictable results, if used in situations with very low reference intensity values.

4. Repeat for all damaged cells, and perform the same analysis on at least two control cells, as described above.

5. Graph normalized intensity values for each measured micro-irradiation event against time to show changes in protein recruitment or type of DNA damage as a function of experimental treatment. 
Representative Results

\section{Characterization of induced DNA damage}

Induction of base lesions and strand breaks is dependent on the laser dose applied to the selected nuclear area and the cellular microenvironment of the cell model used ${ }^{7}$. Fluorescent proteins fused to repair proteins, like XRCC1, 53BP1, Ku70, or Rad51, provide useful single and double strand break markers for establishing the minimal energy required to see accumulation of a fluorescent protein within a damage ROI above the background fluorescence ${ }^{9,19,31}$. Once conditions that induce a response are found, it is critical to characterize the damage mixture induced by that specific wavelength and dose. Attenuation of the dose and duration at the wavelength used can allow the user to minimize the formation of complex damage mixtures. Low laser doses in the UV-A range have been demonstrated to produce predominantly SSBs and a small amount of base lesions, appropriate for studying SSBR and BER pathways ${ }^{10,28}$. Increasing the dose creates more complex base lesions, oxidative and UV induced, and induces more significant numbers of DSBs ${ }^{7,10}$. While the induction of a single species of DNA damage is desirable for examination of specific DNA repair pathways, it is more likely that users are inducing a mixture of DNA lesions, with a specific lesion like SSBs being much more frequent than base lesions or DSBs. This is similar to DNA damage mixtures induced by chemical agents like hydrogen peroxide $\left(\mathrm{H}_{2} \mathrm{O}_{2}\right)$ or methyl methanesulfonate (MMS) ${ }^{32}$. Users need to be aware when they report results that damage mixtures can occur, and careful characterization of the dose and lesions at the induced damage site are necessary to ensure the reproducibility and comparability of their results.

In micro-irradiation studies, XRCC1-GFP is often used as a marker for the induction of base lesions and SSBs ${ }^{9,28}$. XRCC1 is a scaffold protein that plays an important role in SSB repair (SSBR) and base excision repair (BER), and also participates in other repair pathways, like nucleotide excision repair (NER) ${ }^{33,34,35}$. It plays an important coordinating role in DNA repair, interacting with a number of key proteins, including poly(ADPribose) polymerase 1 (PARP-1), DNA polymerase $\beta$ (Pol $\beta$ ), and DNA ligase III. We utilized XRCC1-GFP stably expressed in CHO-K1 cells to determine the laser doses required to generate SSBs and DSBs. We first identified the minimum dose required to induce an observable recruitment of XRCC1-GFP for each wavelength (Figure 1). For the $355 \mathrm{~nm}$ wavelength, a $2 \mathrm{~s}$ dwell time over the defined damage ROI generated an increased fluorescent signal within that ROI, indicating the induction of DNA damage that was detectable over the background (Figure 1A). For $405 \mathrm{~nm}$, an $8 \mathrm{fps}$ scan rate was needed to generate an observable recruitment to the damage ROI (Figure 1A). The dose was then increased ( $10 \mathrm{~s}$ for $355 \mathrm{~nm}$ and $0.5 \mathrm{fps}$ for $405 \mathrm{~nm}$ ) to create a more intense damage ROI (Figure 1A).

Recruitment and retention of XRCC1-GFP at the site of induced damage was then monitored by timelapse imaging. Retention of the protein at the site of DNA damage may indicate on-going DNA repair, while dissociation of the protein from the site of induced damage is often considered a marker for completion of BER or SSBR. However, there has been no clear evidence linking the dissociation of XRCC1 from laser-induced DNA damage sites with the completion of repair. Recruitment of the protein to the site of damage is measured by reporting the mean intensity of the fluorescent signal within the damaged ROI over the mean fluorescent signal measured for the entire nucleus (Figure 1B). This type of normalization helps address intensity fluctuations in the nuclear signal, though other normalization techniques can be employed depending on the cellular distribution of the protein of interest. Here, XRCC1 is localized in the nuclear compartment, so normalization to the nuclear area measures the redistribution of the signal to the damage ROI. The ROI mean fluorescent intensity is then recorded for each image in the timelapse, including the pre-damage image, and graphed as a function of time (Figure 1C).

We then further characterized the damage induced by the two selected laser doses to examine the formation of DNA base lesions. First, formation of CPD, a bulky UV-induced lesion, was probed by immunofluorescence as a marker for NER type lesions (Figure 2A). Then, the oxidatively induced base lesion8-oxodG was probed as a marker for BER type lesions (Figure 2B). No significant increase in CPD lesions were observed at the low dose exposure for both wavelengths ( $2 \mathrm{~s}$ for $355 \mathrm{~nm}$ and $8 \mathrm{fps}$ for $405 \mathrm{~nm}$ ), while the high dose treatment at both wavelengths ( $10 \mathrm{~s}$ for $355 \mathrm{~nm}$ and $0.5 \mathrm{fps}$ for $405 \mathrm{~nm}$ ) did show a significant increase in fluorescent signal observed within the damage ROI (Figure 2A). A scatter plot of the CPD ROI mean intensity for each damaged cell shows heterogeneity in damage formation and detection at both wavelengths and doses, indicating that a low level of CPD lesions may be present at the lower doses, but the load may not be significantly detected until a higher dose is applied. The scatterplot also suggests that inefficiency in antibody detection that may limit accurate quantification of the damage mixtures.

This is further highlighted in the detection of oxidatively induced DNA lesions by the marker 8-oxodG. No clear increase in fluorescent signal within the damage ROI was observed for 8-oxodG at either laser wavelength or dose used (Figure 2B). The antibody used for this work is consistent with previous publications ${ }^{9,10,36}$; however, it should be noted that there can be limitations in observing the formation of 8-oxodG with antibodies ${ }^{37,38}$. Confirmation of the lack of oxidatively induced lesions is also recommended by a second marker, like recruitment of 8 Oxoguanine DNA Glycosylase (OGG1), the enzyme responsible for removal of 8-oxodG from the DNA ${ }^{10}$. We did not observe OGG1 recruitment to our DNA damage sites; however, the formation of low levels of oxidatively induced DNA damage cannot be ruled out completely.

Finally, we examined the formation of DSBs using two markers, $\mathrm{yH} 2 \mathrm{AX}$ and 53BP-1, at the selected laser doses by immunofluorescence (Figure 3 \& 4). $\mathrm{yH} 2 \mathrm{AX}$ is commonly used as a strand break marker, but its specificity for DSBs has been questioned in a number of reports ${ }^{39,40}$ Additionally, it is a phosphorylation event that propagates from the strand break site, so localization of the signal to a strand break can be limited due to this signal propagation. Therefore, combining $\mathrm{YH} 2 \mathrm{AX}$ with $53 \mathrm{BP}-1$ allows for more accurate assessment of DSB formation within the damage ROI.

The response of $\mathrm{YH} 2 \mathrm{AX}$ and 53BP-1 to micro-irradiation is both wavelength and dose dependent. The low dose $(2 \mathrm{~s})$ stimulation at $355 \mathrm{~nm}$ elicits no response at 5 and $20 \mathrm{~min}$, and a weak and variable response $10 \mathrm{~min}$ post irradiation (Figure 3A) for both markers. The high dose (10 s) of $355 \mathrm{~nm}$ micro-irradiation induces an increased fluorescent signal within the damage ROI at 5, 10, and 20 min post irradiation that is reduced at $40 \mathrm{~min}$ (Figure 3B). These results indicate that careful titration of the $355 \mathrm{~nm}$ dose is required to minimize cross-stimulation of repair pathways, as demonstrated by the reduced detection of the double strand break marker $\mathrm{yH} 2 \mathrm{AX}$ at the early time points $(<20 \mathrm{~min})$ at the low dose applied. 
Similar experiments were performed using both low (8 fps) and high dose (0.5 fps) $405 \mathrm{~nm}$ laser stimulation (Figure 4). At this wavelength significant accumulation of fluorescent intensity within the damage ROI was observed for both 53BP-1 and $\mathrm{YH} 2 \mathrm{AX}$ regardless of applied dose indicating that these doses generate a complex mixture of single and double strand breaks almost immediate after the induction of DNA damage (Figure 4). Additionally, the high doses of $405 \mathrm{~nm}$ show an increase in pan-nuclear $\mathrm{YH} 2 \mathrm{AX}$ staining within 10 min of damage induction (Figure 4B, bottom) that makes detection of the damage ROI difficult to report, while the 53BP-1 accumulation is more contained within the damage ROI.

These results clearly demonstrate that $405 \mathrm{~nm}$ micro-irradiation is not appropriate for monitoring SSBR or BER, and that multiple markers for DNA adducts and strand breaks should be employed to fully characterize the induced lesions and DNA repair responses.

\section{Dose-dependent alteration in recruitment and retention of XRCC1-GFP}

Once the induced DNA damage has been characterized, laser micro-irradiation can be an ideal platform for studying the dynamics of DNA repair proteins. The retention and dissociation kinetics of the XRCC1-GFP shows a dose dependency (Figure 1), which is not unexpected given the induction of different damage mixtures by each wavelength. The highest irradiation doses ( $10 \mathrm{~s}$ and $0.5 \mathrm{fps})$ show a higher intensity recruitment of XRCC1-GFP relative to the lower doses and longer retention of the XRCC1-GFP at the site of damage over the 20 min time course (Figure 1C). This indicates that the DNA damage created at higher doses for both 355 and $405 \mathrm{~nm}$ is likely not resolved during the course of the experiment, which is consistent with the appearance and retention of DSB markers, yH2AX and 53BP-1 (Figures 3 \& 4).

Interestingly, the lower damage doses (2 s and $8 \mathrm{fps}$ ) show rapid recruitment of XRCC1-GFP to the damage sites and dissociation of XRCC1GFP over the experimental time course to pre-irradiation levels (Figure 1C). Without the complete characterization of the damage mixture, this may lead to the conclusion that SSBs and base lesions are completely resolved using these damaging conditions. However, the presence of $\mathrm{yH} 2 \mathrm{AX}$ and 53BP-1 at $40 \mathrm{~min}$ for $355 \mathrm{~nm}$ and at $5 \mathrm{~min}$ for the $405 \mathrm{~nm}$ may lead to different interpretations. For the $355 \mathrm{~nm} 2 \mathrm{~s}$ dose, the damage mixture may be predominantly SSBs, so the appearance of DSB markers at 40 min may indicate that some unrepaired lesions may be leading to DSBs or that DSBs generated by this energy are repaired on a longer time scale. Time scale differences between SSBR and DSB repair have been previously reported ${ }^{28,41,42}$. Similarly, the $405 \mathrm{~nm}$ low dose (8 fps) dissociation may indicate a low level of SSBs or a clustering of SSBs that are rapidly converted to DSBs, which has been noted for high damage micro-irradiation and other DNA damaging agents previously ${ }^{43,44,45}$

Together these results highlight the importance of characterizing the induced damage mixtures and utilizing multiple DNA repair proteins and markers for interpreting the recruitment and retention of DNA repair proteins at sites of induced damage.

A)

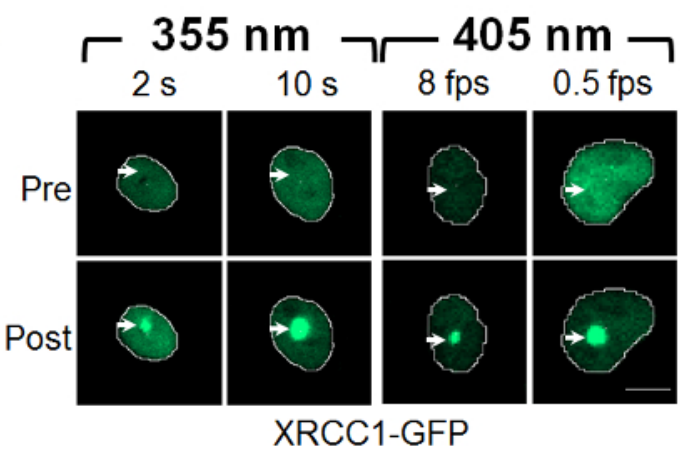

B)

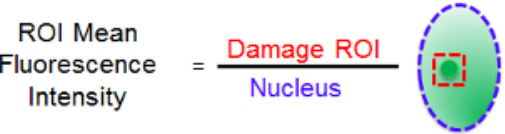

C)

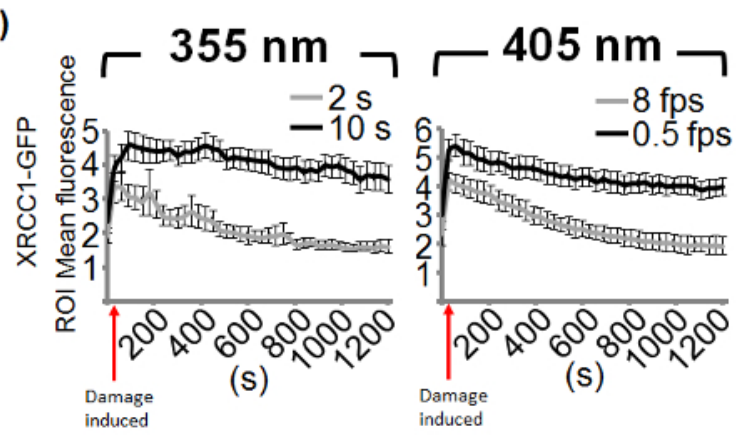

Figure 1. Laser micro-irradiation induces recruitment of XRCC1-GFP.

(A) CHO-K1 cells stably expressing XRCC1-GFP were irradiated and imaged before and immediately after damage induction. Arrows indicate location of micro-irradiation dose and scale bar is $10 \mu \mathrm{m}$. (B) Recruitment is measured by determining the mean fluorescent intensity within the damage ROI and normalizing to the mean fluorescent intensity of the entire nucleus. (C) Dynamics of recruitment can be measured for each timelapse image.Graphs are representative of two independent experiments with error bars representing the SEM ( $n=24)$. Please click here to view a larger version of this figure. 

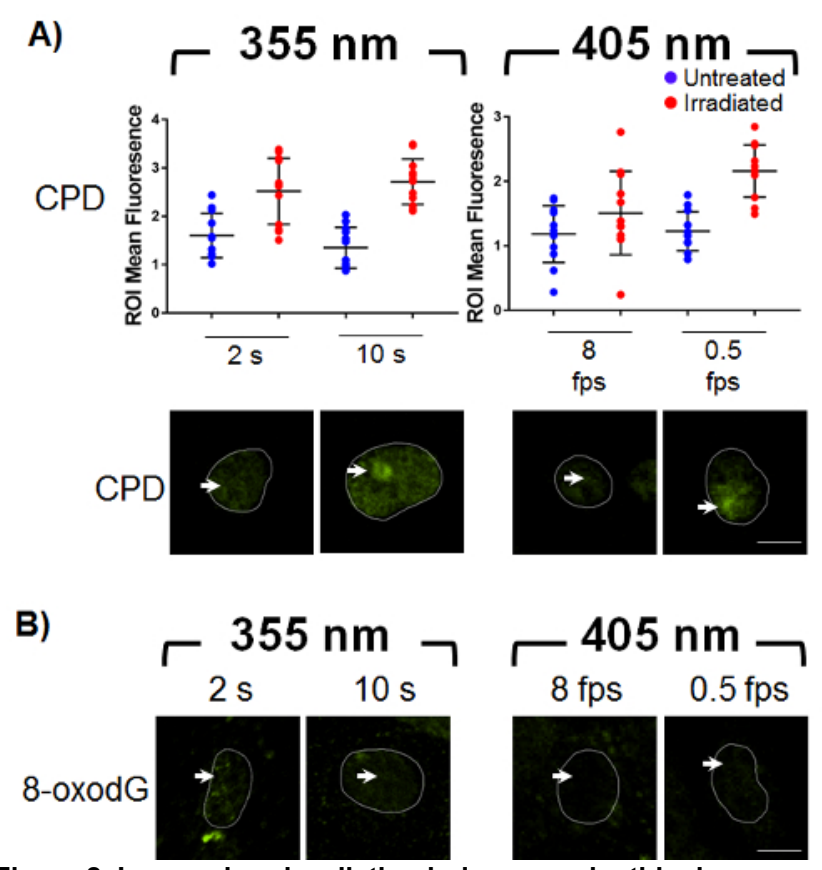

Figure 2. Laser micro-irradiation induces nucleotide damage.

$\mathrm{CHO}-\mathrm{K} 1$ cells were subjected to laser micro-irradiation and fixed immediately after damage induction. Immunofluorescence was performed to detect CPD and 8-oxodG adducts. (A) Top, scatter plot of the ROI mean fluorescence intensities observed in damaged cells after CPD staining. Bottom, representative images of CPD staining. Arrows indicate location of micro-irradiation and the scale bar is $10 \mu \mathrm{m}(\mathrm{n}=12)$. (B)

Representative images for 8-oxodG staining. Arrows indicate location of micro-irradiation and the scale bar is $10 \mu \mathrm{m}$. Please click here to view a larger version of this figure. 

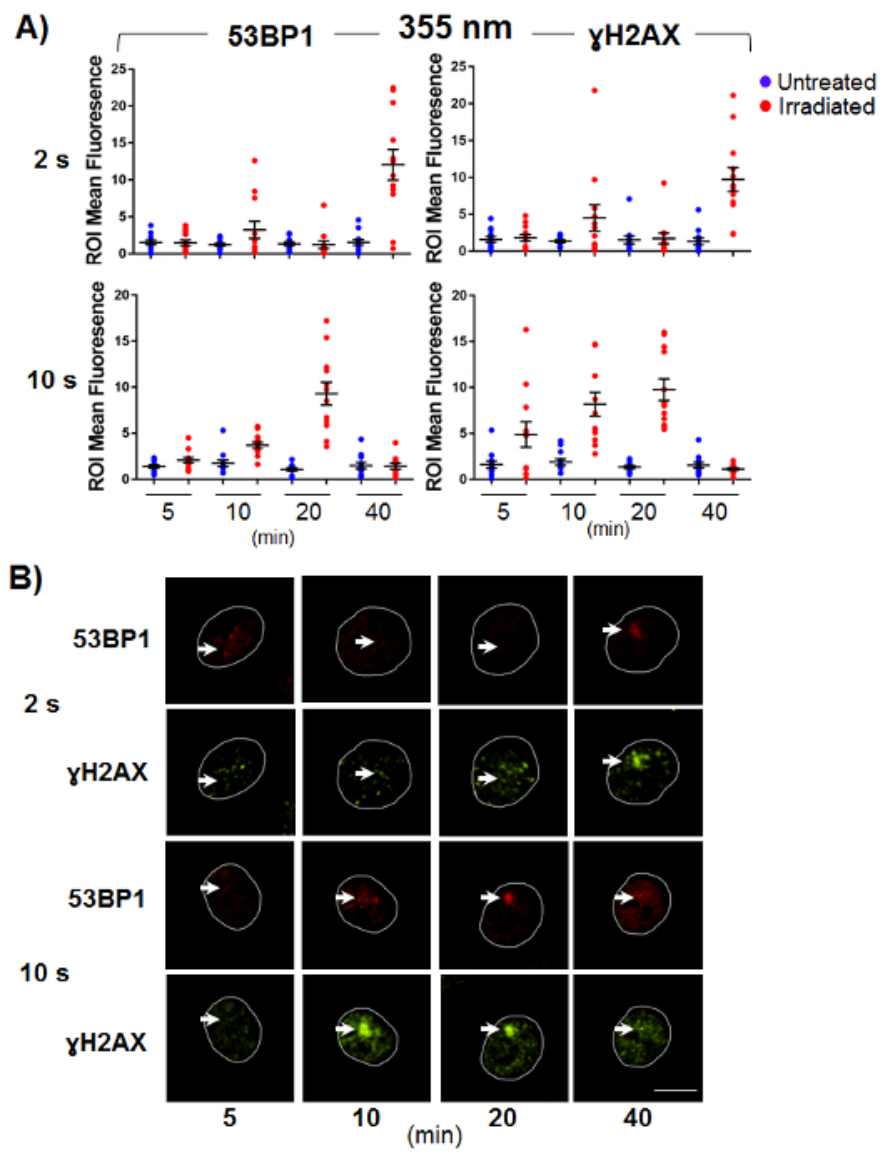

Figure 3. DNA double strand break markers respond to $355 \mathrm{~nm}$ micro-irradiation in a dose dependent manner.

$\mathrm{CHO}-\mathrm{K} 1$ cells were subjected to micro-irradiation and fixed at the time points indicated post-stimulation. Immunofluorescence for the DSB

markers $\mathrm{YH} 2 \mathrm{AX}$ and 53BP-1 was performed. (A) Scatter plot of normalized damage ROI mean fluorescence intensities measured for undamaged and damaged cells. Error bars are representative of the SEM $(n=12)$. (B) Representative images for $y H 2 A X$ and $53 B P-1$ staining. Arrows indicate location of micro-irradiation and the scale bar is $10 \mu \mathrm{m}$. Please click here to view a larger version of this figure. 

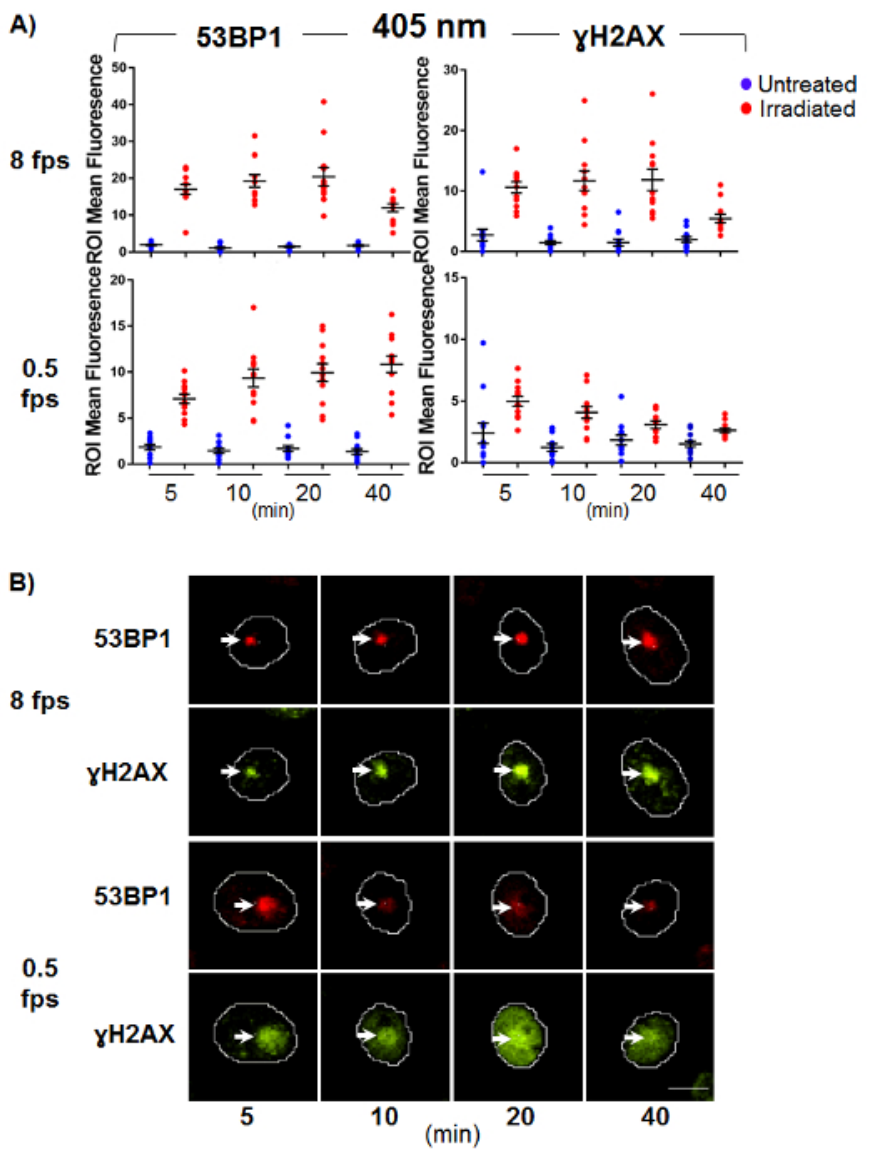

Figure 4. DNA double strand break markers robustly respond to $405 \mathrm{~nm}$ micro-irradiation.

$\mathrm{CHO}-\mathrm{K} 1$ cells were subjected to micro-irradiation and fixed at the time points indicated post stimulation. Immunofluorescence for the DSB markers $\mathrm{YH} 2 \mathrm{AX}$ and 53BP-1. (A) Scatter plot of normalized damage ROI mean fluorescence intensities measured for undamaged and damaged cells. Error bars are representative of the SEM $(n=12)$. (B) Representative images for $\mathrm{YH} 2 \mathrm{AX}$ and 53BP-1 staining. Arrows indicate location of micro-irradiation and the scale bar is $10 \mu \mathrm{m}$. Please click here to view a larger version of this figure.

\section{Discussion}

Using the conditions described here, any confocal microscope with a UV-A or $405 \mathrm{~nm}$ laser, either integrated by the manufacturer or added by the end user, can induce DNA damage within the nucleus of a cell and allow the recruitment of DNA repair proteins to the site of induced DNA damage to be monitored. However, as noted in the protocol and representative results, wavelength selection, applied power, and damage characterization are all important issues that must be addressed first by the user in order to study a specific DNA repair pathway. Additionally, there are other considerations in experimental design that must also be considered by users.

In order to monitor a protein's behavior in live cells post micro-irradiation, a fluorescently labeled protein must be created. The availability of a wide range of fluorescent proteins that can be spectrally separated offers tools for creating protein fusions that can be used for characterizing cellular responses to the induction of DNA damage. Transient transfection of fluorescent proteins of interest allows for quick screening of damaging conditions, mutations, or even inhibitors, while stably transfected cells offer more control over expression levels and allow for other biochemical characterizations to be performed, validating micro-irradiation results. Spectrally distinct fluorescent proteins can also be utilized in the same cell to monitor interactions between proteins within the same pathway or in different pathways. Fluorescent proteins also eliminate the need for specific antibodies for each protein of interest and allow live cell monitoring of protein behavior for long periods of time after the induction of damage.

However, the use of fluorescent proteins also has drawbacks. Without genetic backgrounds deficient in the protein(s) of interest, endogenous proteins will compete with the fluorescently tagged proteins. Conjugation of the fluorescent tag to the $\mathrm{N}$ or $\mathrm{C}$ terminus of the protein may alter protein folding, hinder key protein-protein interactions, or block translocation signals; altering function and potentially impacting recruitment dynamics. These effects have been demonstrated in a number of reports where immunofluorescent staining demonstrated dramatically different recruitment and retention times for endogenous proteins at the damage site ${ }^{28}$. Use of transient transfection or stable clones can also alter the response observed, typically through variations in protein expression levels. Further, the use of multiple fluorescent proteins in a single experiment may also change the dynamics of recruitment, if protein levels are not equilibrated well or if recruitment of the larger fluorescentlytagged protein blocks the recruitment of other proteins. Finally, fluorescent proteins can act as weak sensitization agents, increasing the formation of reactive oxygen species, and potentially altering the DNA damage mixtures induced ${ }^{46,47}$. Despite these drawbacks, fluorescent 
proteins offer a number of distinct advantages in studying DNA repair with micro-irradiation, and if users incorporate appropriate controls, such as the damage characterization described here, they can provide new insight into damage response and protein-protein interactions ${ }^{3}$.

If live cell imaging is not required, immunofluorescence can be used to monitor the response to induced damage. By fixing cells at specific times post damage induction and staining with antibodies specific for proteins and lesions of interest, static snapshots of damage induction and the recruitment and retention of repair proteins can be built. Antibodies can be used to monitor the recruitment of multiple proteins of interest and/or post-translational modifications induced by the DNA damage response. Use of immunofluorescence eliminates the need for fluorescent proteins, and allows for endogenous protein behavior to be examined. However, this method too has its drawbacks. Highly specific antibodies are necessary, and permeabilization and blocking procedures need to be optimized to allow for detection of recruitment with sufficient signal intensity. Fixing procedures are not instantaneous, and this physical constraint limits the temporal resolution of this approach. Mapping the site of damage induction so cells can be re-located after staining can also present significant challenges. The confocal microscope used in this work allows for image-based registration as described above, so damaged cells can be relocated with high precision. If stage registration or etched coverglass is not available, the time investment involved in relocating cells without stage registration, coupled with the inherent delay between damage induction and the imaged recruitment, may make immunofluorescence unattractive to some users. However, the most accurate and complete micro-irradiation experimental designs will incorporate these types of approaches in parallel with the use of fluorescent proteins, as described in the presented protocol.

Here, both live cell imaging and immunofluorescence is used to demonstrate the utility of laser micro-irradiation. Immunofluorescent staining allows us to accurately examine the mixture of DNA damage created and the recruitment of proteins induced by each laser power, which allow us to better interpret the observed alterations in the recruitment and retention of XRCC1-GFP. Based on these results, the use of $405 \mathrm{~nm}$ lasers should be limited for examination of BER and SSBR proteins. Further, the optimal experimental design would include power measurements after the objective, a full damage mixture characterization for each cell line used, and validation of the recruitment and retention observed in fluorescently tagged proteins with immunofluorescence. Obviously, equipment, time, and cost considerations may make these optimal experimental designs impossible for some users. However, the importance of each of these elements is demonstrated here, and users should keep these considerations in mind when beginning micro-irradiation experiments.

\section{Disclosures}

The authors have nothing to disclose.

\section{Acknowledgements}

The authors would like to thank Dr. Samuel H. Wilson at the National Institute of Environmental Health Sciences for the cell lines used in this work.

\section{References}

1. Luijsterburg, M. S. et al. Stochastic and reversible assembly of a multiprotein DNA repair complex ensures accurate target site recognition and efficient repair. J Cell Biol. 189 (3), 445-463 (2010).

2. Vermeulen, W. Dynamics of mammalian NER proteins. DNA Repair (Amst). 10 (7), 760-771 (2011).

3. Berns, M. W. A history of laser scissors (microbeams). Methods Cell Biol. 82 1-58 (2007).

4. Cremer, C., Cremer, T., Zorn, C., \& Schoeller, L. Effects of laser uv-microirradiation (lambda $=2573 \mathrm{~A})$ on proliferation of Chinese hamster cells. Radiat Res. 66 (1), 106-121 (1976).

5. Cremer, C., Cremer, T., Fukuda, M., \& Nakanishi, K. Detection of laser--UV microirradiation-induced DNA photolesions by immunofluorescent staining. Hum Genet. 54 (1), 107-110 (1980).

6. Walter, J., Cremer, T., Miyagawa, K., \& Tashiro, S. A new system for laser-UVA-microirradiation of living cells. J Microsc. 209 (Pt 2), 71-75 (2003).

7. Kielbassa, C., Roza, L., \& Epe, B. Wavelength dependence of oxidative DNA damage induced by UV and visible light. Carcinogenesis. 18 (4), 811-816 (1997).

8. Kielbassa, C., \& Epe, B. DNA damage induced by ultraviolet and visible light and its wavelength dependence. Methods Enzymol. $\mathbf{3 1 9}$ 436-445 (2000).

9. Kong, X. et al. Comparative analysis of different laser systems to study cellular responses to DNA damage in mammalian cells. Nucleic Acids Res. 37 (9), e68 (2009).

10. Lan, L. et al. In situ analysis of repair processes for oxidative DNA damage in mammalian cells. Proc Natl Acad Sci U S A. 101 (38), 13738-13743 (2004).

11. Krasin, F., \& Hutchinson, F. Strand breaks and alkali-labile bonds induced by ultraviolet light in DNA with 5-bromouracil in vivo. Biophys J. 24 (3), 657-664 (1978).

12. Krasin, F., \& Hutchinson, F. Double-strand breaks from single photochemical events in DNA containing 5-bromouracil. Biophys J. 24 (3), 645-656 (1978).

13. Rosenstein, B. S., Setlow, R. B., \& Ahmed, F. E. Use of the dye Hoechst 33258 in a modification of the bromodeoxyuridine photolysis technique for the analysis of DNA repair. Photochem Photobiol. 31 (3), 215-222 (1980).

14. Cremer, T., Peterson, S. P., Cremer, C., \& Berns, M. W. Laser microirradiation of Chinese hamster cells at wavelength $365 \mathrm{~nm}$ : effects of psoralen and caffeine. Radiat Res. 85 (3), 529-543 (1981).

15. Limoli, C. L., \& Ward, J. F. A new method for introducing double-strand breaks into cellular DNA. Radiat Res. 134 (2), $160-169$ (1993).

16. Carlsson, K., Mossberg, K., Helm, P. J., \& Philip, J. Use of UV excitation in confocal laser scanning fluorescence microscopy. Micron and Microscopica Acta. 23 (4), 413-428 (1992).

17. Stelzer, E. H. K., \& Haar, F.-M. Confocal Microscopy: Recent Developments. Advances in Imaging and Electron Physics. 106 293-345 (1999). 
18. Rogakou, E. P., Boon, C., Redon, C., \& Bonner, W. M. Megabase chromatin domains involved in DNA double-strand breaks in vivo. J Cell Biol. 146 (5), 905-916 (1999).

19. Tashiro, S., Walter, J., Shinohara, A., Kamada, N., \& Cremer, T. Rad51 accumulation at sites of DNA damage and in postreplicative chromatin. J Cell Biol. 150 (2), 283-291 (2000).

20. Gassman, N. R., Stefanick, D. F., Kedar, P. S., Horton, J. K., \& Wilson, S. H. Hyperactivation of PARP triggers nonhomologous end-joining in repair-deficient mouse fibroblasts. PLoS One. 7 (11), e49301 (2012).

21. Bolin, C. et al. The impact of cyclin-dependent kinase 5 depletion on poly(ADP-ribose) polymerase activity and responses to radiation. Cell Mol Life Sci. 69 (6), 951-962 (2012).

22. Godon, C. et al. PARP inhibition versus PARP-1 silencing: different outcomes in terms of single-strand break repair and radiation susceptibility. Nucleic Acids Res. 36 (13), 4454-4464 (2008).

23. Hanssen-Bauer, A. et al. XRCC1 coordinates disparate responses and multiprotein repair complexes depending on the nature and context of the DNA damage. Environ Mol Mutagen. 52 (8), 623-635 (2011).

24. Mortusewicz, O., Amé, J. C., Schreiber, V., \& Leonhardt, H. Feedback-regulated poly(ADP-ribosyl)ation by PARP-1 is required for rapid response to DNA damage in living cells. Nucleic Acids Res. 35 (22), 7665-7675 (2007).

25. Mortusewicz, O., Leonhardt, H., \& Cardoso, M. C. Spatiotemporal dynamics of regulatory protein recruitment at DNA damage sites. $J$ Cell Biochem. 104 (5), 1562-1569 (2008).

26. Mortusewicz, O., Rothbauer, U., Cardoso, M. C., \& Leonhardt, H. Differential recruitment of DNA Ligase I and III to DNA repair sites. Nucleic Acids Res. 34 (12), 3523-3532 (2006).

27. Solarczyk, K. J., Zarębski, M., \& Dobrucki, J. W. Inducing local DNA damage by visible light to study chromatin repair. DNA Repair (Amst). 11 (12), 996-1002 (2012).

28. Gassman, N. R., \& Wilson, S. H. Micro-irradiation tools to visualize base excision repair and single-strand break repair. DNA Repair (Amst). $3152-63$ (2015).

29. Botchway, S. W., Reynolds, P., Parker, A. W., \& O'Neill, P. Use of near infrared femtosecond lasers as sub-micron radiation microbeam for cell DNA damage and repair studies. Mutat Res. 704 (1-3), 38-44 (2010).

30. Ferrando-May, E. et al. Highlighting the DNA damage response with ultrashort laser pulses in the near infrared and kinetic modeling. Front Genet. 4135 (2013).

31. Lan, L. et al. Accumulation of Werner protein at DNA double-strand breaks in human cells. J Cell Sci. 118 (Pt 18), $4153-4162$ (2005).

32. Salmon, T. B., Evert, B. A., Song, B., \& Doetsch, P. W. Biological consequences of oxidative stress-induced DNA damage in Saccharomyces cerevisiae. Nucleic Acids Res. 32 (12), 3712-3723 (2004).

33. Caldecott, K. W. DNA single-strand break repair. Exp Cell Res. 329 (1), 2-8 (2014).

34. London, R. E. The structural basis of XRCC1-mediated DNA repair. DNA Repair (Amst). 30 90-103 (2015).

35. Moser, J. et al. Sealing of chromosomal DNA nicks during nucleotide excision repair requires XRCC1 and DNA ligase III alpha in a cell-cyclespecific manner. Mol Cell. 27 (2), 311-323 (2007).

36. Campalans, A. et al. Distinct spatiotemporal patterns and PARP dependence of XRCC1 recruitment to single-strand break and base excision repair. Nucleic Acids Res. 41 (5), 3115-3129 (2013)

37. Cooke, M. S., \& Lunec, J. Immunochemical detection of oxidative DNA damage. Vol. I 275-293 World Scientific, (2003).

38. Rossner, P., Jr., \& Sram, R.J. Immunochemical detection of oxidatively damaged DNA. Free Radic Res. 46 (4), $492-522$ (2012).

39. Cleaver, J. E., Feeney, L., \& Revet, I. Phosphorylated H2Ax is not an unambiguous marker for DNA double-strand breaks. Cell Cycle. 10 (19), 3223-3224 (2011).

40. Rybak, P. et al. Low level phosphorylation of histone $\mathrm{H} 2 \mathrm{AX}$ on serine 139 ( $\mathrm{yH} 2 \mathrm{AX}$ ) is not associated with DNA double-strand breaks. Oncotarget. 7 (31), 49574-49587 (2016).

41. Haince, J. F. et al. PARP1-dependent kinetics of recruitment of MRE11 and NBS1 proteins to multiple DNA damage sites. J Biol Chem. 283 (2), 1197-1208 (2008)

42. Caldecott, K. W. Single-strand break repair and genetic disease. Nat Rev Genet. 9 (8), 619-631 (2008).

43. Lomax, M. E., Gulston, M. K., \& O'Neill, P. Chemical aspects of clustered DNA damage induction by ionising radiation. Radiat Prot Dosimetry. $99(1-4), 63-68$ (2002).

44. Ma, W., Westmoreland, J. W., Gordenin, D. A., \& Resnick, M. A. Alkylation base damage is converted into repairable double-strand breaks and complex intermediates in G2 cells lacking AP endonuclease. PLoS Genet. 7 (4), e1002059 (2011).

45. Siddiqi, M. A., \& Bothe, E. Single- and double-strand break formation in DNA irradiated in aqueous solution: dependence on dose and OH radical scavenger concentration. Radiat Res. 112 (3), 449-463 (1987).

46. Sano, Y., Watanabe, W., \& Matsunaga, S. Chromophore-assisted laser inactivation--towards a spatiotemporal-functional analysis of proteins, and the ablation of chromatin, organelle and cell function. J Cell Sci. 127 (Pt 8), 1621-1629 (2014).

47. Wojtovich, A. P., \& Foster, T. H. Optogenetic control of ROS production. Redox Biol. 2 368-376 (2014). 